\title{
Role of MRI Imaging and MR Spectroscopy in the Diagnosis of Ocular Hydatid Cyst: Case Report
}

\author{
Kishor Taori, Amit Disawal, Jawahar Rathod, Anand Hatgaonkar, Suresh Dhakate, \\ Vishal Bakare, Prasad Wavare, Rakhi Puria \\ Department of Radio-Diagnosis, Government Medical College and Hospital, Nagpur, India \\ Email: kishortaori@gmail.com
}

Received December 6, 2012; revised January 18, 2013; accepted January 29, 2013

\begin{abstract}
Hydatid disease is one of the common parasitic infestations in many parts of the world with involvement of any organ inside the human body. Ocular hydatid cyst is one of such locations and is a relatively rare entity. However, with the help of MRI imaging and super added benefit of MRI Spectroscopy, the diagnosis of hydatid cyst can be reached to with great confidence, which dictates the proper line of management in such patients.
\end{abstract}

Keywords: Ocular; Hydatid Cyst; MRI Imaging; MR Spectroscopy; Pyruvate Peak

\section{Introduction}

Hydatid disease is an endemic parasitic disease in many parts of the world and is well described in literature [1]. The most common form being the hepatic and the lung hydatid cyst [1]. Orbital Hydatid cyst is rare manifestation of echinococcal infestation. It represents $1 \%-2 \%$ of all hydatid disease and less than $0.3 \%-1 \%$ of all intraorbital lesions, however, the percentage is quite high in some endemic regions of the world [2-7]. Here, we report a case of orbital hydatid cyst diagnosed by MRI findings in correlation with MRI Spectroscopy and confirmed by post-operative histopathological analysis.

\section{Case Report}

A 48 years old male patient came was referred with complaints of progressive painless proptosis and mild restriction of eye movements involving the left eyeball since 3 months. There was no significant reduction in visual acuity. On clinical examination, there was lid edema and chemosis noted on local examination of the eye. The patient was farmer by occupation and had positive history of contact with domestic animals for many years. There was no history of any trauma to the eye.

Initially, the patient was evaluated by B scan of the eye which showed extra-ocular anechoic well-defined cystic lesion on the superior aspect of the left eyeball (Figure 1). The lesion was suspicious to be hydatid cyst and for further evaluation MRI orbit was done. 1.5 T MRI scan was done with surface coil which is basically a receiver coil.
MRI of the orbit revealed well-defined cystic lesion, appearing hypointense on T1W sequences (Figure 2), hyper intense on T2W sequences (Figure 3) and hypo intense on T2W FLAIR sequence (Figure 4), supero-medially involving the extra-ocular but intra and extra-conal compartment of the left orbit. It was measuring approximately $2.1 \times 2.4 \times 1.9 \mathrm{~cm}$ with mild peripheral enhancement of its walls (Figure 5) and there was no significant peri-lesional edema. There was no focus of blooming noted inside the lesion on gradient echo sequences (Figure 6) ruling out the possibility of hemorrhagic component or calcifications. Also, diffusion weighted images and apparent diffusion coefficient maps did not show signific ant diffusion restriction within the lesion ruling out abscess formation. The optic nerve had been displaced with remodeling of the bony orbital roof and the lateral wall.

To confirm the nature of the cystic fluid inside the lesion, single voxel proton MRI Spectroscopy of the lesion with short TE (31 milliseconds) was performed (Figure 7). After using water suppression adjustment and baseline correction, peak integrals were calculated. It revealed significant Pyruvate peak at $2.4 \mathrm{ppm}$ and elevated lactate seen as a doublet peak at $1.3 \mathrm{ppm}$. As this was an orbital lesion, NAA concentration did not came into picture, which is basically a marker of neuronal integrity. Pyruvate peak is more specific and has been mentioned in literature as an in vivo marker of hydatid infestation.

The patient was screened for other primary focus of hydatid cyst inside the ultrasound (US) of the abdomen, which did not reveal any other lesion. 


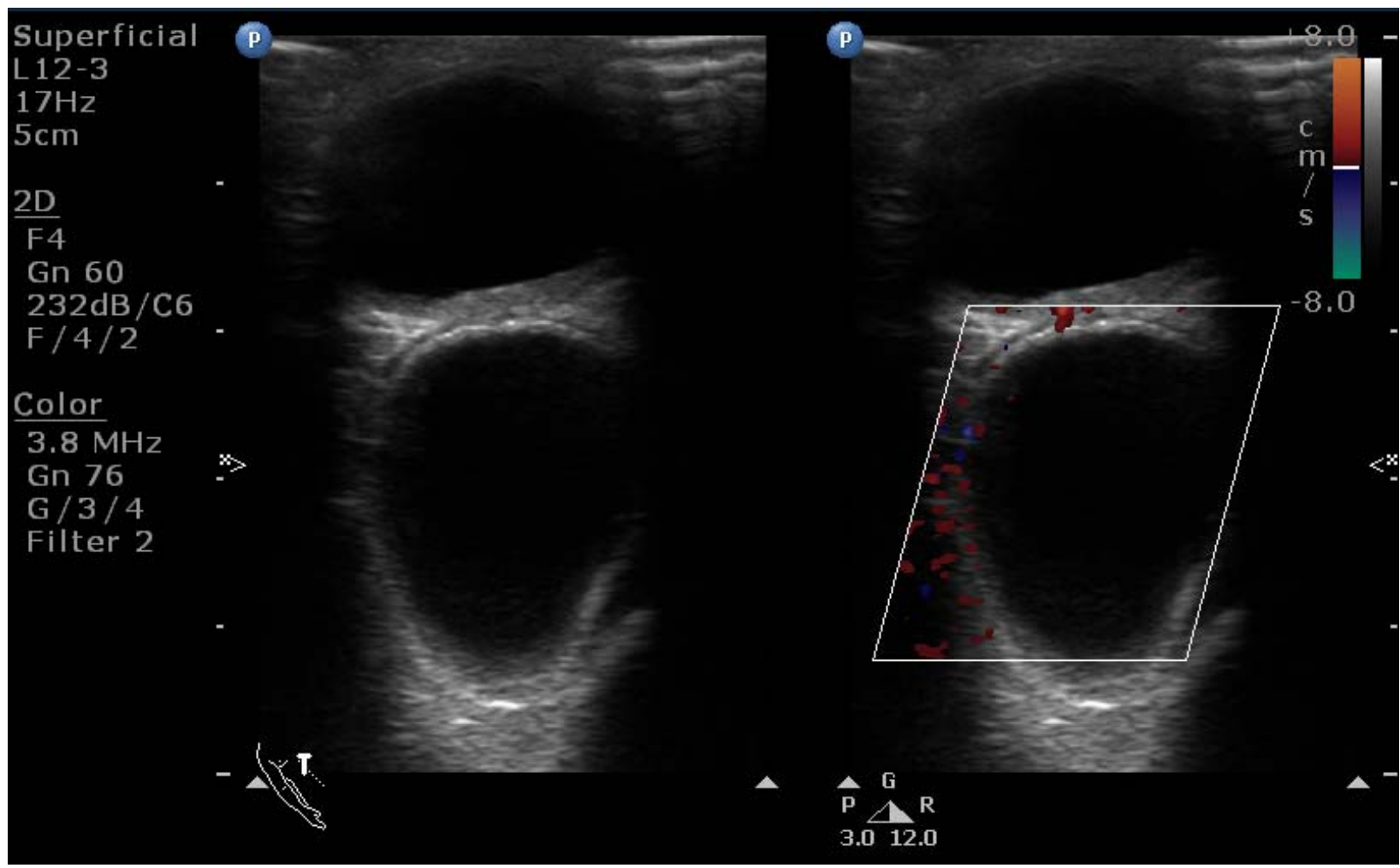

Figure 1. Ultrasonography image showing well defined retro-globar, cystic, anechoic and avascular lesion compression the left eyeball.

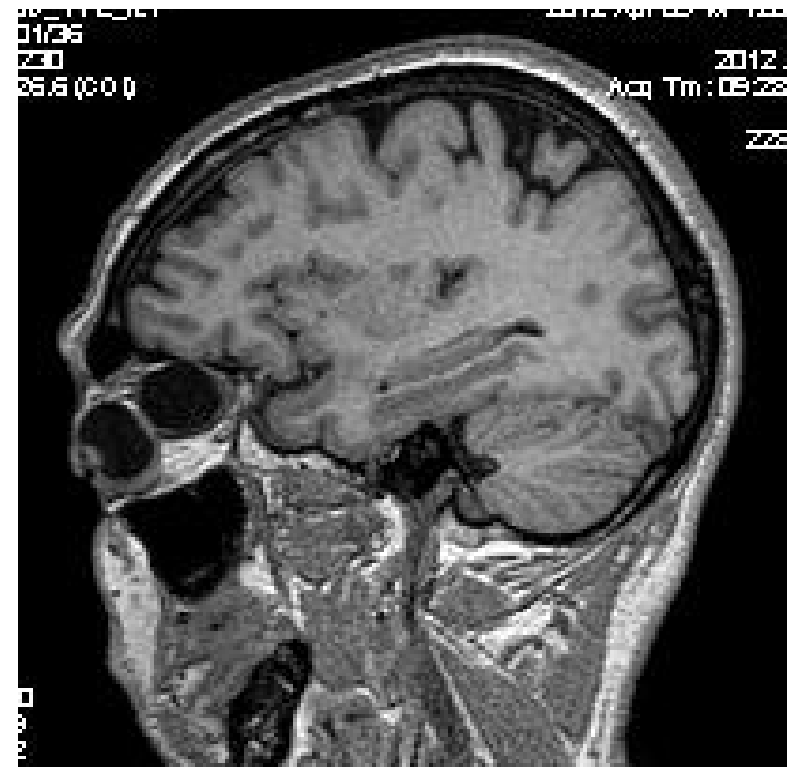

Figure 2. T1W sagital image showing the uni-locular hypo intense cystic lesion noted postero-superior to the left eyeball.

After starting the patient on Albendazole, the decision for surgical removal of the cyst was taken. By frontoorbital craniotomy approach and excision of the orbital roof, the cyst was accessed. The contents were aspirated and then the cyst walls excised. Povidone iodine solution

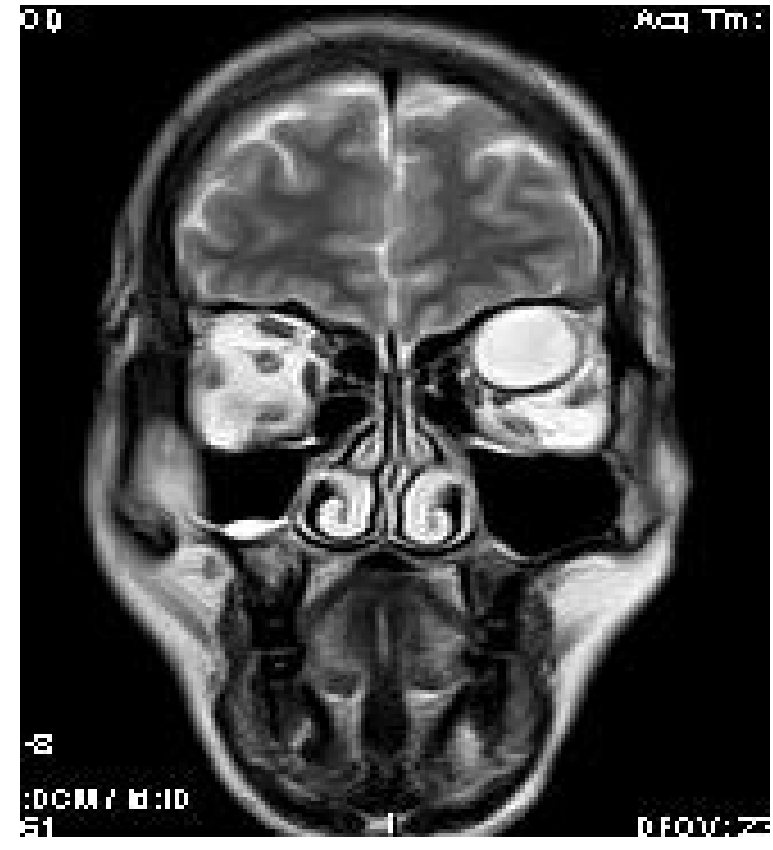

Figure 3. T2W coronal image showing the intra and extra-conal components of the lesion on left side.

$(5 \% \mathrm{w} / \mathrm{v})$ was introduced inside the residual cavity which acts as a scolicidal agent. The surgery was uneventful and specimen sent for histo-pathological examination which confirmed the diagnosis of infected hydatid cyst. 


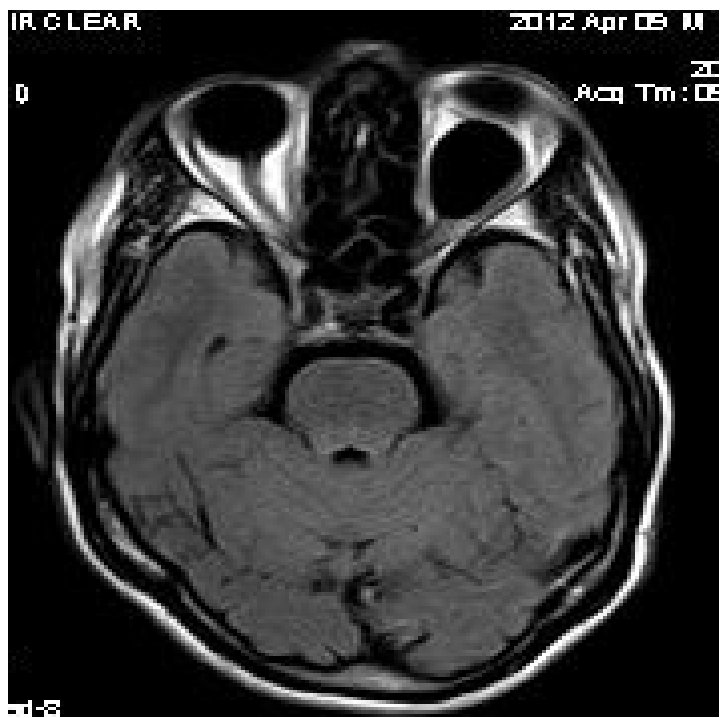

Figure 4. Axial T2W FLAIR image showing well-defined hypointense lesion in left orbit with suppression of the fluid signal intensity.
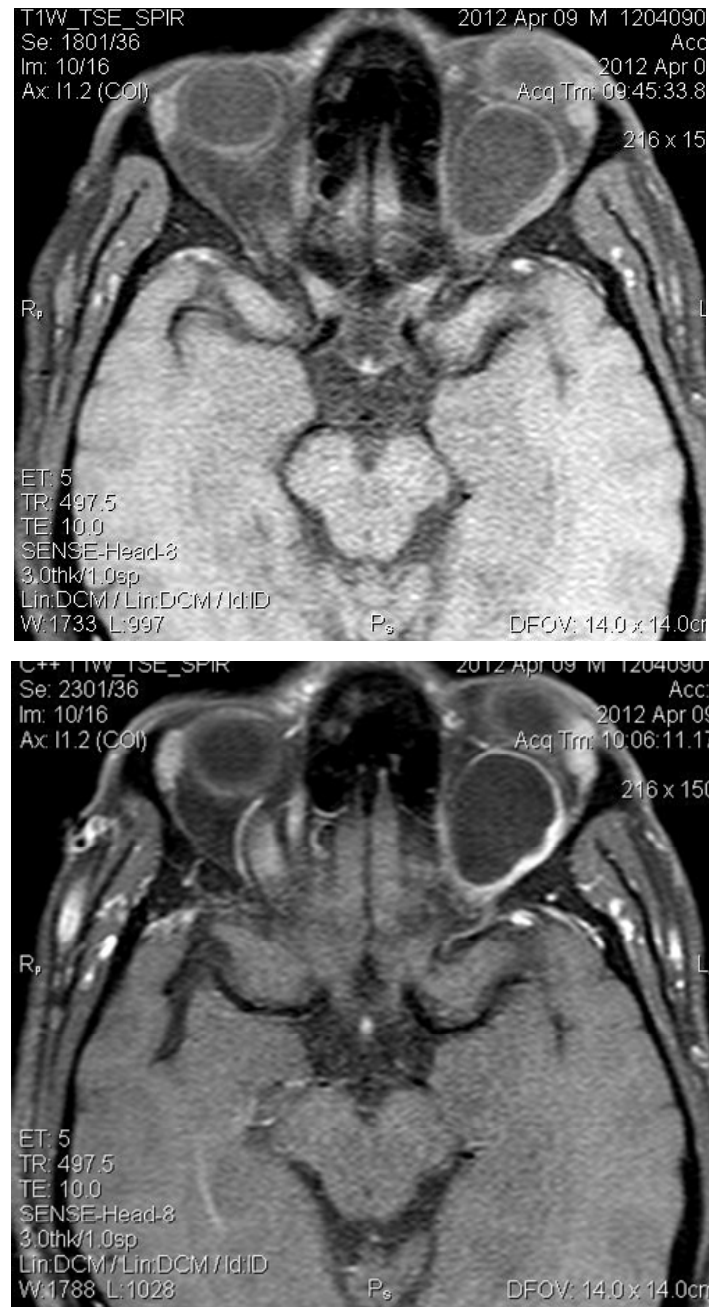

Figure 5. Pre and post contrast T1W fat suppressed axial images showing mild peripheral enhancement of the lesion.

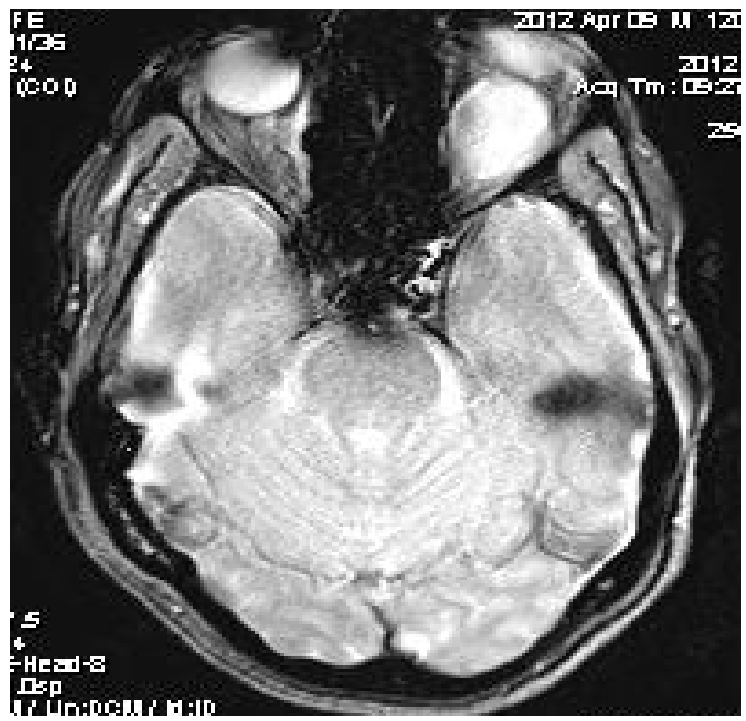

Figure 6. Gradient echo based image showing no significant blooming within the lesion ruling out hemorrhage or calcification.

\section{Discussion}

The parasitic agent for Hydatid disease is Echinoccocus granulosus, with dog being the primary host and sheep and cattles as intermediate host. Man is an accidental host, in this life cycle. Most commonly liver and lungs are involved [1]. It may be unilocular or multilocular. Orbital hydatid is rare manifestation of hydatid disease, and represents $0.3 \%-1 \%$ of all cases [2-7]. Orbital hydatid can be primary or secondary depending on whether it is the only manifestation or a part of disseminated hydatidosis [8,9]. Deve (1921) and Demaria (1919) successfully demonstrated the development of a hydatid cyst experimentally in the eyes of experimental animals. Orbital hydatid cysts are usually located on the supero-lateral or supero-medial aspect of the orbit [10].

Patients present commonly with painless proptosis for months, associated with mild periorbital pain and mechanical restriction of ocular movements. Defective vision, chemosis, exposure keratitis, headache are other clinical features [11].

The differential diagnosis of such ocular cysts includes abscess, orbital hematoma, lymphangioma, lacrimal cysts or dermoid cysts. Abscess can be suspected if constitutional symptoms like fever, headache is present. Only Peripheral enhancement rules out other lesions like hemangioma, lymphangioma and malignant tumors which have their own unique enhancement patterns. Dermoid cysts show fatty and calcified components within simultaneously.

The various radiological investigations including ultrasound (US), CT scan \& MRI in correlation with MRI Spectroscopy help to confirm the correct diagnosis. On 


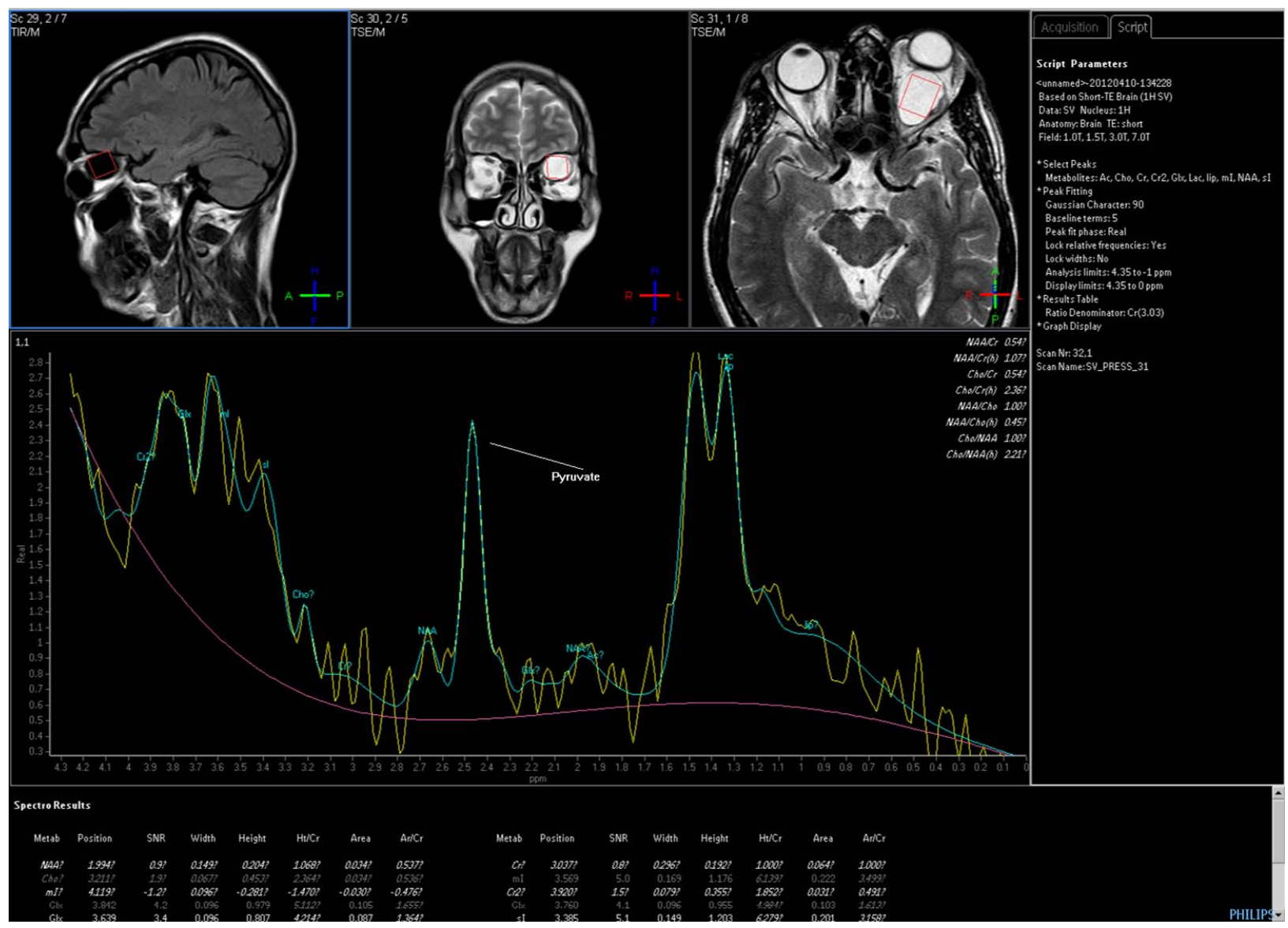

Figure 7. Single voxel MRI spectroscopy image with short TE of 31 milliseconds showing Pyruvate and Lactate peaks at 2.4 and $1.3 \mathrm{ppm}$ respectively.

US, diagnostic double layer sign of the cyst wall is seen in some cases. "Spoke wheel pattern" and "water lily sign" are classically described in literature. Contrast enhanced CT scan shows mildly enhancing cystic lesion having fluid attenuation of 3 - $30 \mathrm{HU}$ with few calcifications, multiple septae and globe deformity if present. MRI shows well defined lesion, T1 and T2 FLAIR hypointense \& T2 hyper intense lesion, characteristic of cystic lesion.

MRI Spectroscopy is a new tool for confirmatory diagnosis of the hydatid cyst in cases of dilemma. Proton MR Spectroscopy shows markedly elevated pyruvate, elevated succinate, acetate, and alanine peaks [12-14], and lipid-lactate peak at $1.33 \mathrm{ppm}$. Elevated myoinositol has been reported by Sreedhar et al. [13]. There are pyruvate and succinate peaks found at $2.4 \mathrm{ppm}$ and $2.5 \mathrm{ppm}$ respectively. These are metabolic end products arising from microorganisms. Succinate peak is, however, nonspecific and can occur in hydatid disease, neurocysticercosis, and abscesses [14]. Pyruvate peak is more specific and has been mentioned as an in vivo marker of hydatid infestation [13-16]. Acetate and succinate peaks are seen in both parasitic cysts and bacterial abscesses and are noted at $1.9 \mathrm{ppm}$ and at $2.5 \mathrm{ppm}$ respectively. Abscesses show higher Acetate:succinate ratio, while succinate:acetate ratio is higher in hydatid and neurocysticercosis [12, 14]. Monika et al., have shown creatine peak in neurocysticercosis and its absence in hydatid [17]. Garg et al. experimented on fertility assessment of hydatid cyst exvivo by MR spectroscopy [12], which showed presence of malate and fumarate in addition to other resonances in fertile cysts. Zoran et al. described unusual appearance of the cyst on MRI which can be attributed to the layering of hydatid sand [18]. In this patient, MR Spectroscopy with short TE, there was presence of lactate peak, pyruvate peak, with small acetate peak at $1.9 \mathrm{ppm}$. The succinate and pyruvate peaks are close together and cannot be easily distinguished from each other.

Total surgical excision is the definitive treatment of such lesions. Various surgical approaches have been described. There are two main routes, the transcranial approach which is selected for lesions having an intracranial extension when it is necessary to expose the optic nerve and for lesions located superiorly to the optic nerve and the lateral orbital approach is the procedure most commonly used to treat intraorbital lesions located in the 
lateral compartment of the orbit. Rupture of the cyst during surgical dissection is common occurrence and hence scolicidal agent has to be used to prevent relapse. Albendazole is usually begun 2 - 4 weeks before surgery as an adjuvant and also decreases relapse.

\section{Conclusion}

In conclusion, MRI scans in correlation with MRI Spectroscopy can confidently diagnose Hydatid cyst due to its unique characteristic findings, which helps in deciding the proper line of management. MRI Spectroscopy has evolved as a modern non invasive modality of choice for diagnostic purposes which is based on the grounds of analyzing the biochemical alterations inside the body due to various pathological conditions.

\section{REFERENCES}

[1] J. J. Plorde and P. G. Ramsey, "Nematodes, Cestodes, and Hermaphroditic Trematodes," In: J. D. Wilson, E. Braunwald, K. J. Isselbacher, et al., Eds, Harrison's Principles of Internal Medicine, 12th Edition, McGraw-Hill Inc., New York, 1991, pp. 827-828.

[2] A. Shah, M. V. Kirtane, C. E. D'Souza, et al., "Hydatid Cyst of the Orbit (A Case Report)," Journal of Postgraduate Medicine, Vol. 34, 1998, pp. 43A-44A.

[3] H. Talib, "Orbital Hydatid Disease in Iraq," British Journal of Surgery, Vol. 59, No. 5, 1972, pp. 391-394. doi:10.1002/bjs. 1800590517

[4] A. Gomez Morales, J. O. Croxatto, L. Crovetto, et al., "Hydatid Cysts of the Orbit. A Review of 35 Cases," Ophthalmology, Vol. 95, No. 8, 1988, pp. 1027-1032.

[5] X. An and X. Y. Cheng, "Hydatid Cysts of the Orbit in Xinjiang: A Review of 18 Cases," Orbit, Vol. 18, No. 3, 1999, pp. 151-155. doi:10.1076/orbi.18.3.151.2705

[6] V. Klauss and H. S. Chana, "Ocular Tumors in Africa," Social Science \& Medicine, Vol. 17, No. 22, 1983, pp. 1743-1750. doi:10.1016/0277-9536(83)90386-6

[7] M. E. Jimenenez-Mejias, J. C. Alarcon-Cruz, F. J. Marquez-Rivas, et al., "Orbital Hydatid Cyst: Treatment and Prevention of Recurrences with Albendazole plus Praziquantel," Journal of Infection, Vol. 41, No. 1, 2000, pp. 105-107. doi:10.1053/jinf.2000.0687

[8] O. Staindl and C. Kenkel, "Throat, Nose and Ear Medicine," NHO Host Network Operator HNO Harvard News
Office HNO Helvetica Narrow Oblique, 1985, pp. 404408.

[9] S. M. Betharia, N. Pushker, V. Sharma, et al., "Disseminated Hydatid Disease Involving Orbit, Spleen, Lung and Liver," Ophthalmologica, Vol. 216, 2002, pp. 300-304. doi:10.1159/000063839

[10] H. B. Diren, H. Ozcanli, M. Boluk, et al., "Unilocular Orbital, Cerebral and Intraventricular Hydatid Cysts: CT Diagnosis," Neuroradiology, Vol. 35, No. 2, 1993, pp. 149-151. doi:10.1007/BF00593974

[11] C. Gokcek, N. Bayar and Z. Buharai, "Total Removal of an Unruptured Orbital Hydatid Cyst," Canadian Journal of Ophthalmology, Vol. 36, No. 4, 2001, pp. 218-220.

[12] M. Garg and R. K. Gupta, "MR Spectroscopy in Intracranial Infection," In: J. Gillard, A. Waldman and P. Barker, Eds., Clinical MR Neuroimaging, Cambridge University Press, Cambridge, 2005, pp. 380-406.

[13] M. Sreedhar, "Disseminated Hydatid Disease: A Case Report with MR Spectroscopic Correlation," Indian Journal of Radiology and Imaging, Vol. 16, No. 4, 2006, pp. 771774. doi:10.4103/0971-3026.32344

[14] K. Chand, A. K. Kanodia, G. Manpreet and A. Neeraj, "In Vivo Proton Magnetic Resonance Spectroscopy in a Known Case of Intracranial Hydatid Cyst," Neurology India, Vol. 53, No. 3, 2005, pp. 337-338. doi:10.4103/0028-3886.16937

[15] P. N. Jay Kumar, S. G. Srikanth, H. S. Chandrasekhar, J. M. Kovoor, S. K. Shankar and B. Anandh, "Pyruvate: An in Vivo Marker of Cestodal Infection in Human Brain on Proton MR Spectroscopy," Journal of Magnetic Resonance Imaging, Vol. 18, No. 6, 2003, pp. 675-680. doi:10.1002/jmri.10409

[16] A. Kohli, R. K. Gupta, H. Poptani and R. Roy, "In Vivo Proton Magnetic Resonance Spectroscopy in a Case of Intracranial Hydatid Cyst," Neurology, Vol. 45, No. 3, 1995, pp. 562-564. doi:10.1212/WNL.45.3.562

[17] M. Garg, S. Chawla, K. N. Prasad, R. Roy, S. S. Sikora, R. Kumar, et al., "Differentiation of Hydatid Cyst from Cysticercus Cyst by Proton MR Spectroscopy," NMR in Biomedicine, Vol. 15, No. 5, 2002, pp. 320-326. doi: $10.1002 / \mathrm{nbm} .774$

[18] Z. Rumboldt, H. Jednacak, J. Talan-Hraniloviל, T. Rumboldt and M. Kalousek, "An Unusual Appearance of Cisternal Hydatid Cyst," American Journal of Neuroradiology, Vol. 24, No. 1, 2003, pp. 112-114. 